\title{
Miniaturized Substrate Integrated Waveguide Diplexer Using Open Complementary Split Ring Resonators
}

\author{
Mostafa DANAEIAN, Kambiz AFROOZ, Ahmad HAKIMI \\ Department of Electrical Engineering, Shahid Bahonar University of Kerman, Kerman, Iran \\ mdanaeian@eng.uk.ac.ir, \{afrooz, hakimi\}@uk.ac.ir \\ Submitted July 5, 2016 / Accepted December 22, 2016
}

\begin{abstract}
In this paper, two miniaturized planar diplexers based on the substrate integrated waveguide (SIW) structure loaded by open complementary split-ring resonators (OCSRRs) are proposed. The working principle is based on the theory of evanescent mode propagation. The proposed SIW diplexers operate below the cutoff frequency of the waveguide. Both the complementary split-ring resonators (CSRRs) and the OCSRRs behave as electric dipoles however, the resonance frequency of the OCSRRs is approximately half of the resonance frequency of the CSRRs. Therefore, the electrical size of the OCSRRs is larger than the CSRRs. Accordingly, the OCSRRs are more appropriate for the SIW miniaturization. At first, the filtering response of the SIW structure loaded by OCSRR unit cells is investigated. Then, two miniaturized SIW diplexers which consist of two cascaded OCSRR unit cells with different orientations are designed. For the first diplexer (Type I), the fractional bandwidths of operation for the up and down channels are $9.52 \%$ and $2.59 \%$ at $4.2 \mathrm{GHz}$ and $5.8 \mathrm{GHz}$, respectively. For the second diplexer (Type II), the fractional bandwidths of operation for the up and down channels are $5.95 \%$ and $2.51 \%$ at $4.7 \mathrm{GHz}$ and $5.6 \mathrm{GHz}$, respectively. Finally, in order to validate the ability of the proposed OCSRR unit cells in the size reduction, two designed diplexers are fabricated and experimental verifications are provided. A good agreement between the results of measurement and simulation is achieved. The proposed diplexers show significant advantages in terms of size reduction, low loss, high isolation, and integration with other planar circuits.
\end{abstract}

\section{Keywords}

Open Complementary Split Ring Resonators (OCSRRs), electric dipoles, substrate integrated waveguide (SIW), evanescent mode, miniaturization

\section{Introduction}

Recently, substrate integrated waveguide (SIW) has already attracted an extreme interest in the design of mi- crowave and millimeter-wave integrated circuits [1-4]. The SIW structure is synthesized on a planar dielectric substrate with linear arrays of metallized via holes or posts connected with two metal plates on the top and bottom sides. This structure has been quickly developed because of its advantages such as high quality factor, low insertion loss, high power-handling capability, low cost, and more importantly, easy integration with planar circuits [1-4].

Diplexers are one of essential components in transceivers for microwave multi-service and multi-band communication systems. Diplexer is a three ports device which is used to separate transmit/receive channels connected to a common antenna. This component provides a high bandisolation between two different output ports to avoid interference between each other. In order to achieve a few desired specifications for a high-performance diplexer in terms of low insertion losses, strong channel rejection and high isolation, this device can be realized by different filter configurations [5-10]. The SIW structures are suitable to realize essential filter elements of the diplexers because of the advantage of these structures. However, these structures suffer from large sizes.

On the other hand, there has been a rising attention in the field of the double negative (DNG) structures which can be realized by resonance structures or non-resonance structures [11-13]. It has been shown by Marques et al. that the resonant-type approach is useful for the synthesis of bandpass filters (BPFs). The essential elements for implementations of the resonant-type approach are the split ring resonators (SRRs) and complementary split ring resonators (CSRRs). These metamaterial resonant elements are frequency-selective structures and the electrical size of them is significantly small. The SRRs provide a negative effective permeability while the CSRRs exhibit an effective negative permittivity in a narrow band at the resonate frequencies [12], [13]. Other types of these resonant particles are the open split ring resonator (OSRR) and the open complementary split ring resonator (OCSRR). The electrical size of the OCSRR is larger than the electrical size of the CSRR. Therefore, this particle can be more appropriate to miniaturize the physical size of microwave components which are implemented by the resonant-type approach [12-13]. 
In this paper, the evanescent-mode technique is used to miniaturize the SIW diplexers. Two compact planar SIW diplexers loaded by the OCSRR unit cells with different orientations are designed. These proposed diplexers are realized by a three-port device with two cascaded SIWOCSRR filters whose working principle is based on the theory of evanescent mode propagation. Therefore, more miniaturization is achieved by using OCSRRs instead of CSRRs. Finally, two proposed SIW diplexers are fabricated and tested. A good agreement between the simulation and measurement's results is obtained. The designed diplexers exhibit some useful characteristics, such as high isolation, low loss and more important, high compactness.

\section{Realization of the SIW-OCSRR Filters}

Several methods and topologies are reported [5-6] in order to miniaturize the SIW filters. Among them, the evanescent-mode technique attracts an extreme attention because of easy etching some components on the top or bottom metal surface of the SIW structure [7-10]. According to this theory, an additional forward passband at the frequency range below the waveguide cutoff could be achieved by loading the electric dipoles on the metal cover of the SIW structure. Since the CSRRs behave as electrical dipoles, so that some miniaturized SIW filters based on this technique have been reported [14]. The passband of the miniaturized filter is located below the cutoff frequency of the SIW structure [14].

The structures and the equivalent-circuit models of the CSRRs and the OCSRRs are shown in Fig. 1(a) and Fig. 1(b), respectively. Both the CSRR and the OCSRR are modeled by means of the shunt-connected resonant tank formed by one capacitance and one inductance. As shown in Fig. 1, the inductance of the CSRR is a quarter of the OCSRR, so the resonance frequency of the CSRR is twice the resonance frequency of the OCSRR with the same footprint. Therefore, the electrical size of the OCSRR is larger than that of the CSRR and more miniaturization could be achieved by loading the OCSRR on the SIW surface [15]. To achieve further miniaturization, the SIW bandpass filters loaded by the OCSRRs have been proposed in [15] where two prototypes of the SIW-OCSRR filters have been designed. The configurations of these filters are depicted in Fig. 2. In order to increase the coupling between the two OCSRRs as well as suppress the propagation of the dominant mode of the SIW structure, the metal strip in the middle of two OCSRRs is removed.

To meet the given specification in the filter design, it is important to follow the classic methodology. In this method, the essential parameters could be defined in terms of the circuit elements of a low-pass prototype filter. These design parameters include the coupling coefficient and external quality factor. To specify the physical configuration of the filters based on this method, the relationship
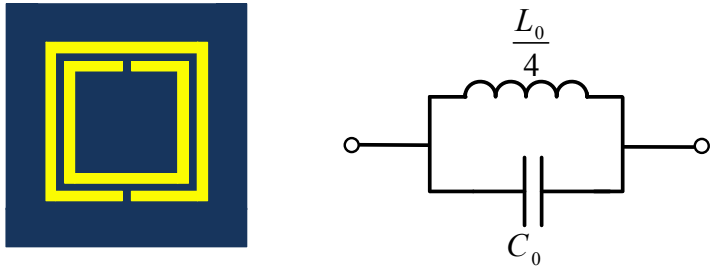

(a)
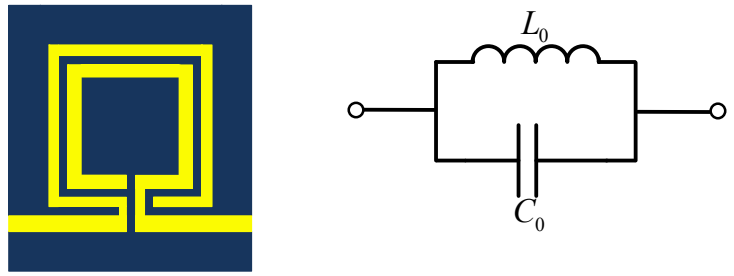

(b)

Fig. 1. Topology and equivalent circuit model of (a) the CSRR and (b) the OCSRR.

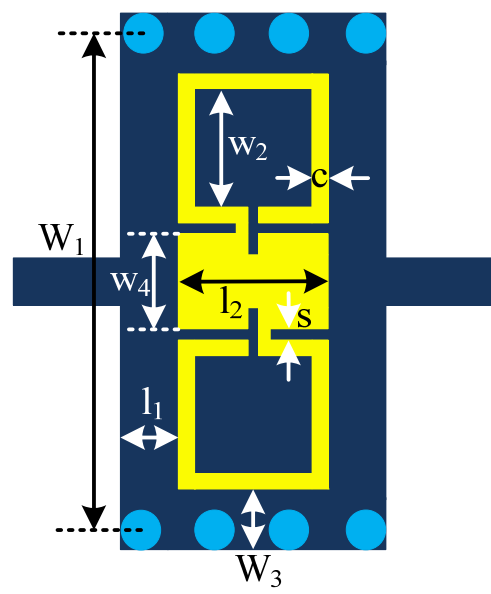

(a)

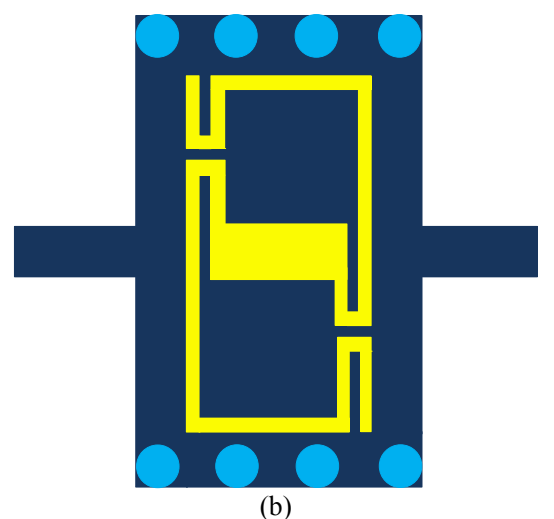

Fig. 2. Configuration of the SIW-OCSRR filters: (a) face-toface oriented (Type I), (b) side-by-side reversely oriented (Type II).

between coupling coefficient, external quality factor and physical structures should be determined. Consequently, the distances $w_{4}, l_{1}$, and $l_{2}$ can be achieved by using their relationship with the coupling coefficient and extracted external quality factor. The external quality factor and coupling coefficient can be evaluated by using the following relations [15]: 


$$
\begin{gathered}
Q_{\mathrm{e}}=\frac{2 f_{0}}{B W}, \\
K=\frac{f_{1}^{2}-f_{2}^{2}}{f_{1}^{2}+f_{2}^{2}}
\end{gathered}
$$

where $f_{0}$ refers to the resonance frequency, $B W$ refers to the $3 \mathrm{~dB}$ bandwidth, and $f_{1}$ and $f_{2}$ stand for the higher and lower resonant frequencies of the coupled resonators. Fig. 3 illustrates the external quality factor and coupling coefficient versus the distances $l_{1}, l_{2}$, and $w_{4}$.

On the other hand, the center frequency of the passbands can be easily moved by resizing the dimension of the OCSRRs which includes the distances $w_{2}, c$, and $s$. The resonance frequency of the OCSRR is achieved from:

$$
f=\frac{1}{2 \pi \sqrt{L_{0} C_{0}}} .
$$

Also, by resizing the length and width of the distances between two OCSRRs (resizing the distances $l_{1}$ and $w_{4}$ ) the bandwidth can be easily adjusted. Figure 4 presents the results from simulation by resizing the distances $l_{1}$ and $w_{4}$. As can be seen in these figures, when the distances $l_{1}$ and $w_{4}$ are smaller, a stronger coupling is obtained which leads to a larger bandwidth.

A current diagram of the SIW-OCSRR filter for two different frequencies (that is, within the forward passband below the cutoff frequency and stopband below the cutoff frequency) is depicted in Fig. 5. At $5.5 \mathrm{GHz}$, the signal propagates and OCSRRs are excited. In this case, forwardwave propagation below the waveguide cutoff is obtained based on the resonant behavior of the OCSRRs. However, at $3 \mathrm{GHz}$, OCSRRs are not excited and the injected power is retuned back. Furthermore, due to the use of slots in the proposed OCSRR structures, these slots may produce radiation in the upper half space. Therefore, the radiation loss could be quantified as follows:

$$
R_{\mathrm{r}}=1-\left|S_{11}\right|^{2}-\left|S_{12}\right|^{2}
$$

The simulated radiation loss is depicted in Fig. 6 which is extracted by using (4) under the conditions of lossless substrate and metal. As we can see from Fig. 6 the radiation loss is less than 0.026 for Type I and 0.032 for Type II which implies that the slots have a little effect on the radiation loss in the filters design.

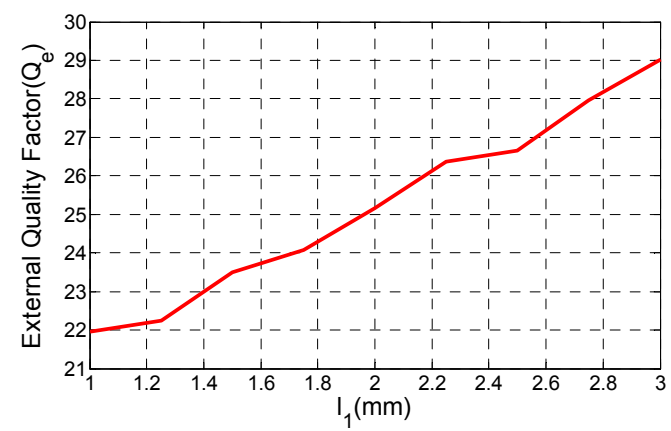

(a)

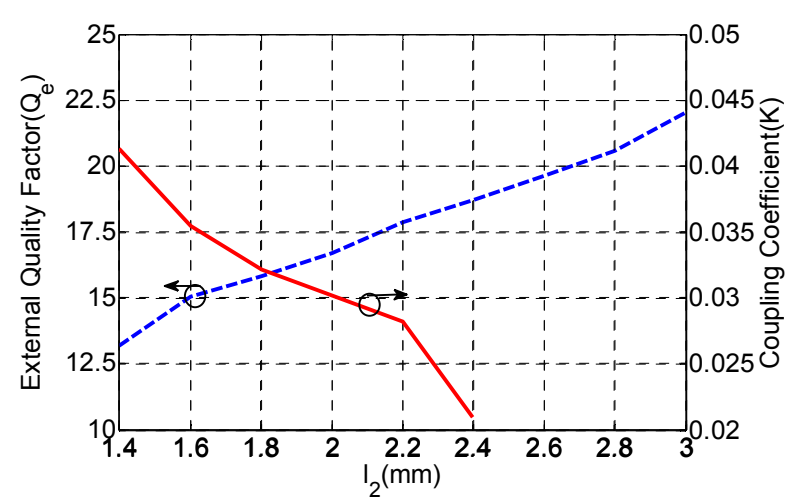

(b)

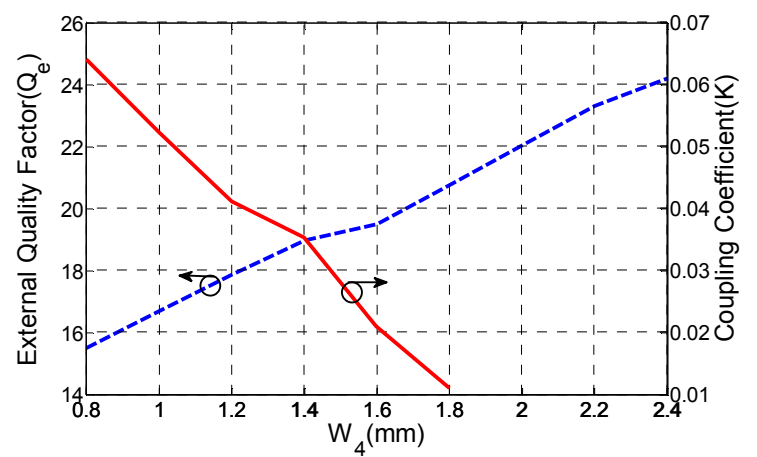

(c)

Fig. 3. (a) External quality factor and coupling coefficient as a function of $l_{1}$. (b) External quality factor as a function of $l_{2}$. (c) External quality factor and coupling coefficient as a function of $W_{4}$.

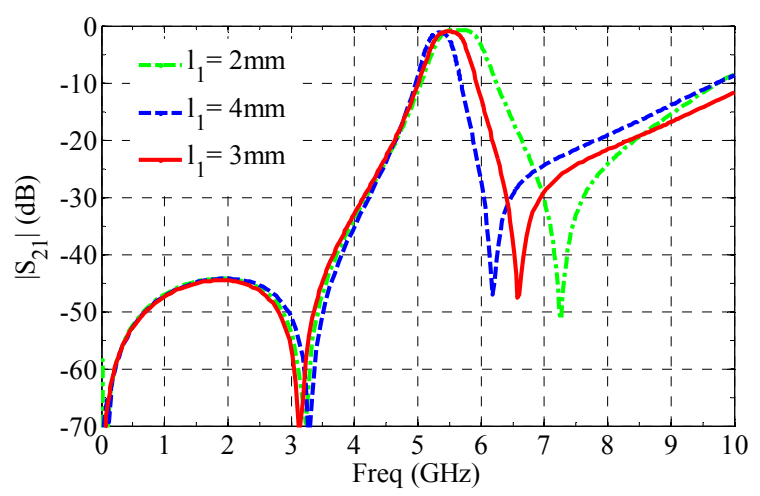

(a)

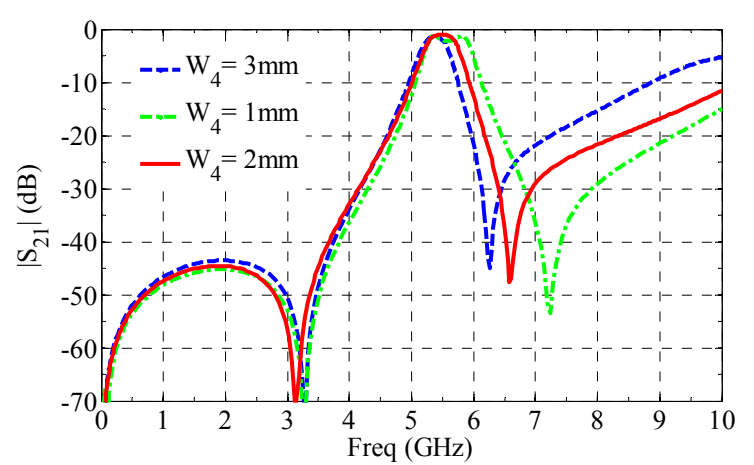

(b)

Fig. 4. (a) Simulated $\left|S_{21}\right|$ in $\mathrm{dB}$ with different $l_{1}$ and (b) simulated $\left|S_{21}\right|$ in $\mathrm{dB}$ with different $W_{4}$. 


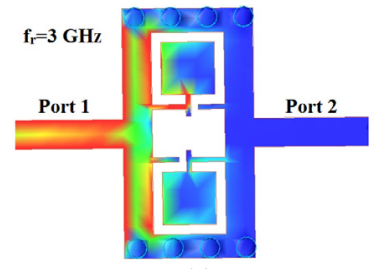

(a)

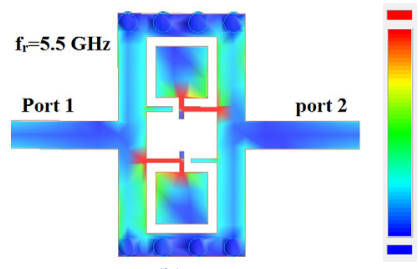

(b)
Fig. 5. Current diagrams for the SIW-OCSRR filter at two different frequencies.

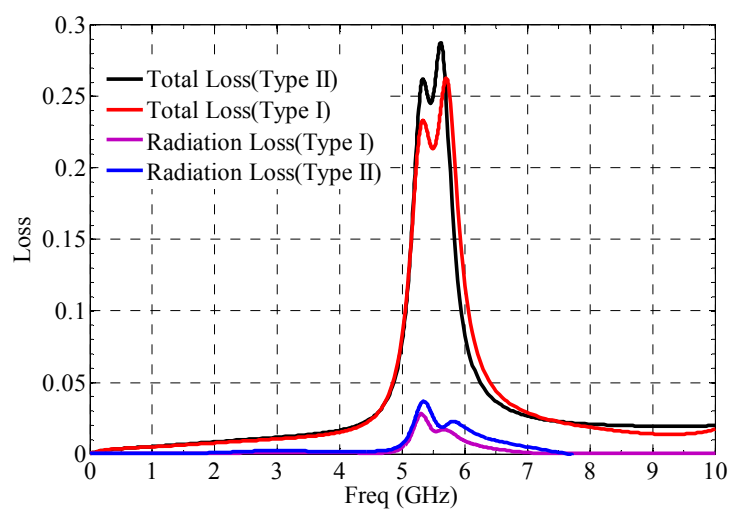

Fig. 6. Calculated radiation loss and total transmission losses (radiation loss + dielectric loss + conductor loss) of the proposed SIW-OCSRR.

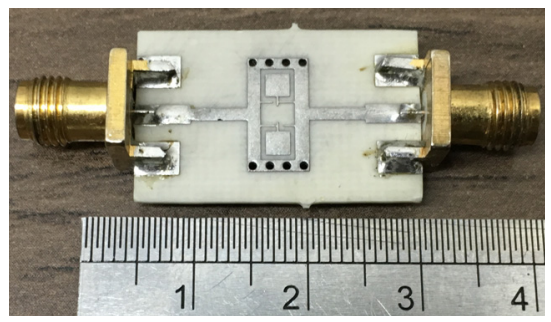

(a)

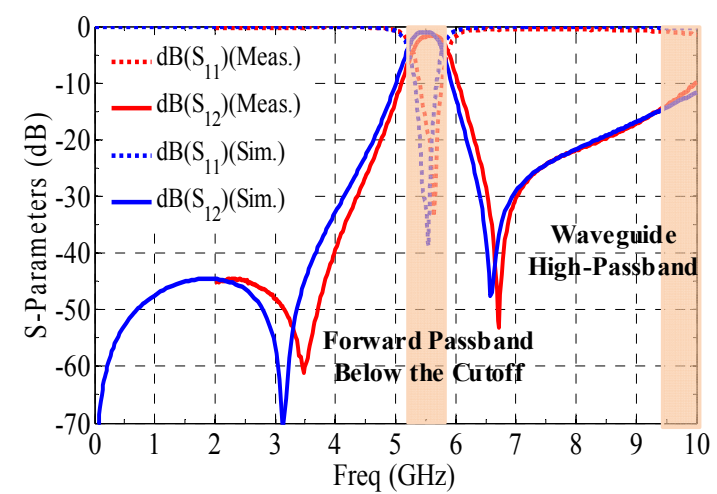

(b)

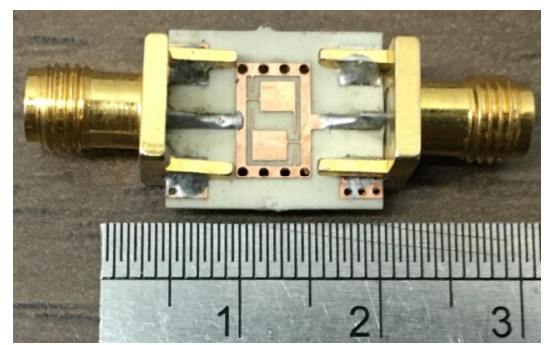

(c)

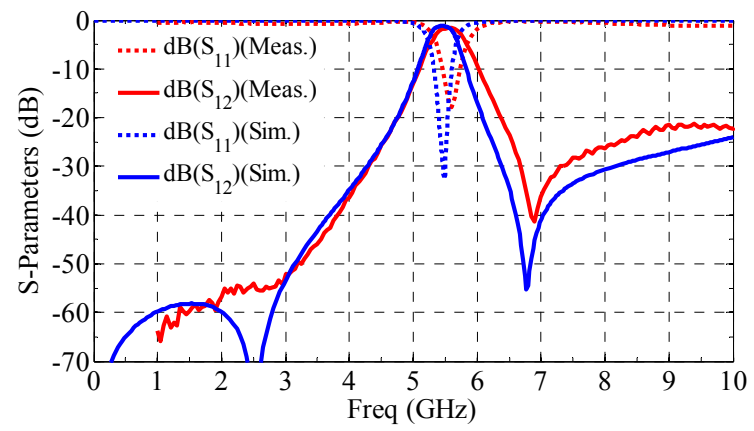

(d)

Fig. 7. Photographs, simulated and measured frequency responses of the fabricated SIW-OCSRR filters: (a) Type I, (b) Type II [15].

Also, in Fig. 6, a comparison by including all losses (which contain radiation loss + dielectric loss + conductor loss) for these resonator is presented which finally leads to $1.2 \mathrm{~dB}$ insertion loss for Type $\mathrm{I}$ and $1.6 \mathrm{~dB}$ insertion loss for Type II.

Figure 7 shows the photograph, simulated and measured transmission responses of the SIW-OCSRR filters which demonstrates that the designed filters have very small size compared with similar filters. As can be seen in Fig. 7 (b) and (d), a forward passband below the waveguide cutoff at center frequency of $5.5 \mathrm{GHz}$ is achieved which is good for WLAN applications [15].

\section{Design Procedure of the Proposed SIW Diplexers}

Configurations of the proposed diplexers with their physical parameters are depicted in Fig. 8. Figure 8(a) shows the first layout of the proposed diplexer with a pair of OCSRRs in the face-to-face orientation referred to as Type I. The second configuration of the proposed diplexer with a pair of OCSRRs in the side-by-side reverse orientation is referred to as Type II and is shown in Fig. 8(b). The proposed diplexers consist of two SIW channel filters loaded by OCSRRs and three $50 \Omega$ SIW-microstrip transitions. Three $50 \Omega$ microstrip feed lines have been used here for the purpose of measurement where these microstrip feed lines provide the pads for the attachment of the edge launch SMA connectors.

The channel SIW-OCSRR filters play an essential role in designing the proposed diplexers in which a transmitter and a receiver filter are cascaded together. These proposed diplexers have been achieved after designing two SIW filters with appropriate features. The frequency responses of the proposed diplexers are shown in Fig. 9. The frequency responses of the proposed diplexers which are realized based on SIW-OCSRR filters are simulated by using 3-D electromagnetic simulator. In the simulations, the metallic and dielectric loss have been taken into account by using the conductivity of copper $\sigma=$ $5.8 \times 10^{7} \mathrm{~S} / \mathrm{m}$ and the loss tangent $\tan \delta=0.0027$ for the substrate. As shown in Fig. 9, the simulated insertion losses 


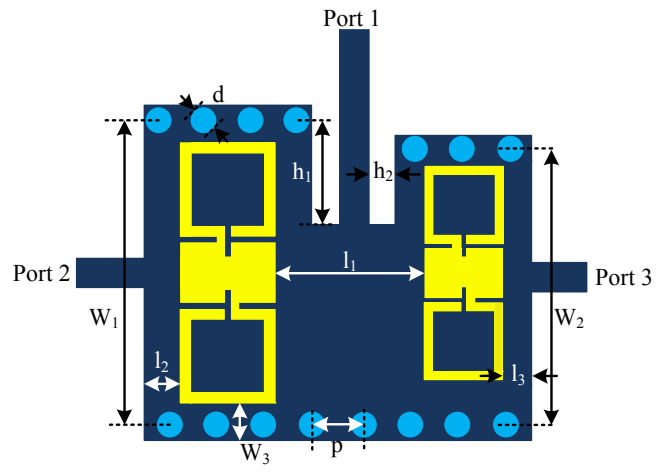

(a)

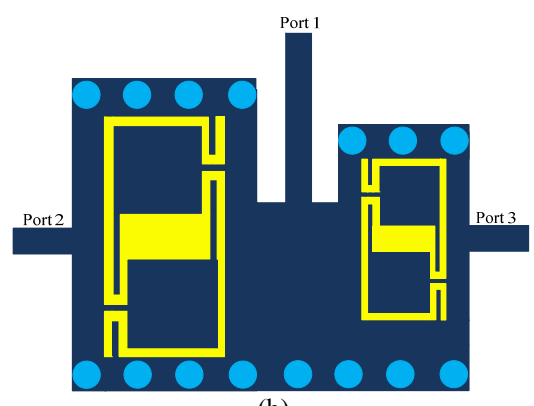

(b)

Fig. 8. Configuration of the proposed SIW-OCSRR diplexers: (a) Type I, (b) Type II.

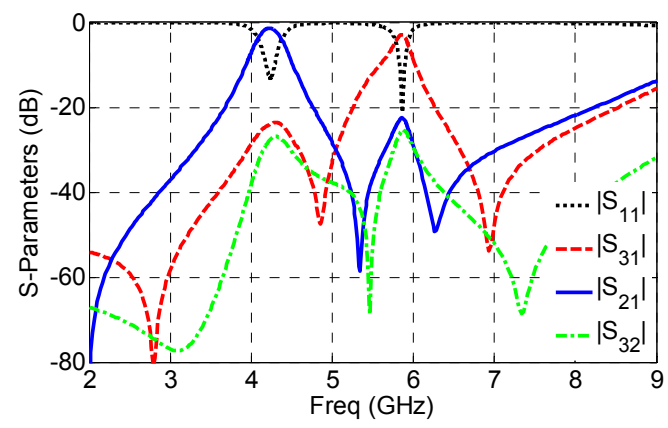

(a)

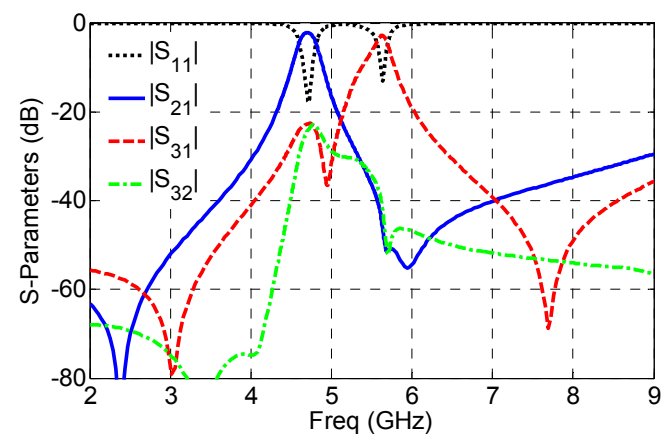

(b)

Fig. 9. Simulated frequency responses and isolation of the proposed SIW diplexers: (a) Type I, (b) Type II.

at the two bands for diplexer Type I are approximately $1.5 \mathrm{~dB}$ and $2.8 \mathrm{~dB}$, respectively and the simulated insertion losses at the two bands for diplexer Type II are approximately $1.7 \mathrm{~dB}$ and $2.1 \mathrm{~dB}$, respectively. The simulated return losses at the lower and higher channels of the proposed diplexer (Type I) are better than $16 \mathrm{~dB}$ and for the proposed diplexer (Type II) arre better than $17 \mathrm{~dB}$. The simulated out-of-band rejection of each channel filter is better than $23 \mathrm{~dB}$ for both diplexers. It can be seen that low in-band insertion loss, high isolation, and high stopband rejection with high selectivity are obtained.

By resizing the dimension of the OCSRRs in the SIW-OCSRR filters and consequently, by changing the center frequency of the channel filters, the two passbands could be very close to each other or away. In order to get a low in-band reflection, we can tune the inset length $h_{1}$, slot width $h_{2}$ and the distance between the feeding and resonators $\left(l_{2}\right.$ and $\left.l_{3}\right)$.

The obtained design dimensions of the proposed diplexer (Type I), which are shown in Fig. 8(a), and the dimensions of the proposed diplexer (Type II), which are shown in Fig. 8(b) are exhibited in Tab. 1 and Tab. 2, respectively. The overall dimensions of the diplexer Type I is less than $0.15 \lambda_{0} \times 0.14 \lambda_{0}(11.4 \mathrm{~mm} \times 10.2 \mathrm{~mm})$ and the total sizes of the diplexer Type II is less than $0.22 \lambda_{0} \times 0.13 \lambda_{0}(14.2 \mathrm{~mm} \times 8.5 \mathrm{~mm})$ where $\lambda_{0}$ is the free space wavelength of the first channel center frequency.

\begin{tabular}{|c|c|c|c|}
\hline$W_{1}=10.2 \mathrm{~mm}$ & $W_{2}=9.2 \mathrm{~mm}$ & $W_{3}=1 \mathrm{~mm}$ & $l_{1}=3.2 \mathrm{~mm}$ \\
\hline$l_{2}=1 \mathrm{~mm}$ & $l_{3}=1 \mathrm{~mm}$ & $h_{1}=3.4 \mathrm{~mm}$ & $h_{2}=0.7 \mathrm{~mm}$ \\
\hline$p=1.5 \mathrm{~mm}$ & $d=0.8 \mathrm{~mm}$ & $c=0.3 \mathrm{~mm}$ & $s=0.2 \mathrm{~mm}$ \\
\hline
\end{tabular}

Tab. 1. Dimensions of the proposed diplexer (Type I).

\begin{tabular}{|c|c|c|c|}
\hline$W_{1}=8.5 \mathrm{~mm}$ & $W_{2}=7.8 \mathrm{~mm}$ & $W_{3}=1 \mathrm{~mm}$ & $l_{1}=3.8 \mathrm{~mm}$ \\
\hline$l_{2}=1 \mathrm{~mm}$ & $l_{3}=0.8 \mathrm{~mm}$ & $h_{1}=3.4 \mathrm{~mm}$ & $h_{2}=0.8 \mathrm{~mm}$ \\
\hline$p=1.5 \mathrm{~mm}$ & $d=0.8 \mathrm{~mm}$ & $c=0.4 \mathrm{~mm}$ & $s=0.2 \mathrm{~mm}$ \\
\hline
\end{tabular}

Tab. 2. Dimensions of the proposed diplexer (Type II).

\section{Fabrication and Measurements}

In order to confirm the simulated results in the previous section, two designed diplexers are fabricated and measured. The two samples are implemented on a Rogers RO4003C substrate with a thickness of $0.508 \mathrm{~mm}$ and relative dielectric constant of 3.55 . Figure 10 shows the measured frequency responses of the proposed diplexers which are measured by the employment of a network analyzer Rohde \& Schwarz, zvk. These results contain the measured reflection and transmission coefficients $\left(\left|S_{11}\right|\right.$, $\left|S_{21}\right|$ and $\left.\left|S_{31}\right|\right)$, as well as the isolation $\left(\left|S_{32}\right|\right)$ for the both diplexers which are measured. It is obvious that the measured results are in a good agreement with the simulation ones. As shown in Fig. 10, the measured insertion losses at $4.33 \mathrm{GHz}$ and $5.99 \mathrm{GHz}$ for diplexer Type I are approximately $1.6 \mathrm{~dB}$ and $3.2 \mathrm{~dB}$, respectively and the measured insertion losses at $4.78 \mathrm{GHz}$ and $5.77 \mathrm{GHz}$ for diplexer Type II are approximately $1.8 \mathrm{~dB}$ and $2.3 \mathrm{~dB}$, respectively.

The measured return losses in the passbands are achieved less than $22 \mathrm{~dB}$ and $15 \mathrm{~dB}$ at lower $(4.33 \mathrm{GHz})$ and higher $(5.99 \mathrm{GHz})$ bands of the Type I diplexer, respectively, and less than $20 \mathrm{~dB}$ and $14 \mathrm{~dB}$ at lower $(4.78 \mathrm{GHz})$ and higher $(5.77 \mathrm{GHz})$ bands for the Type II diplexer, respectively. Similarly, the measured out-of-band 


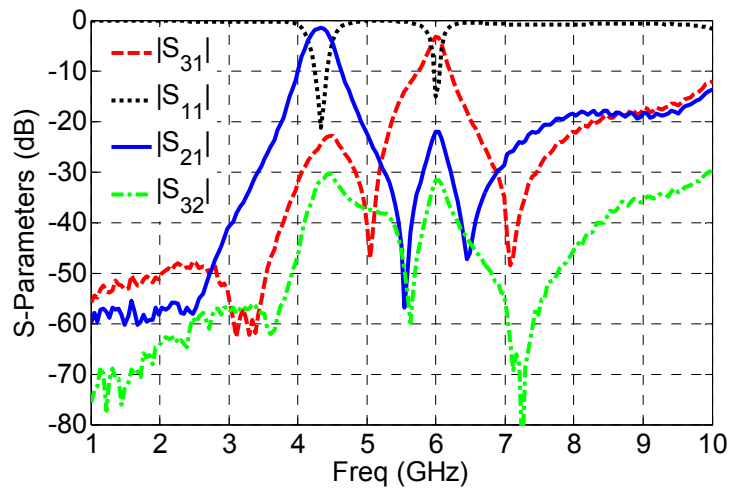

(a)

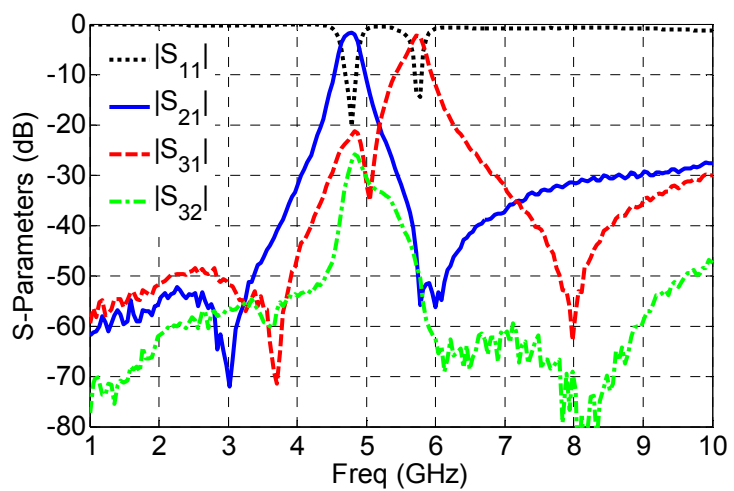

(b)

Fig. 10. Measured frequency responses and isolation of the proposed SIW diplexers: (a) Type I, (b) Type II.

rejection of each channel filter is better than $23 \mathrm{~dB}$ for Type I diplexer and better than $22 \mathrm{~dB}$ for Type II diplexer. Some minor differences between simulation and measurement results may be caused by the extra loss from the SMA connectors, by the limited accuracy of fabrication and measurement. The simulated channel return losses at Port 2 and Port 3 are also plotted in Fig. 11. As shown in these figures, the simulated return losses in both channels for both diplexers are better than $18.2 \mathrm{~dB}$. Here both proposed diplexers work in C-band based on two different channel filters. However, by changing the center frequency of the channel filters which is discussed above, the proposed diplexers can operate in different band like S-band and Cband or C-band and X-band. Generally, the wave propagation at these frequencies for the SIW structure loaded by OCSRRs still keeps the manner of $\mathrm{TE}_{10}$ mode. These particles at the cutoff frequency of the SIW structure are not resonant and do not affect the initial propagation of the SIW structures [14].

Figure 12 shows the group delay for both channels of the Type I and Type II diplexers, respectively, which are flat. In the passband, the group delay from input port 1 to output port 2 with the center frequency $4.33 \mathrm{GHz}$ is less than $1.65 \mathrm{~ns}$ and the group delay from input port 1 to output port 3 with the center frequency $5.99 \mathrm{GHz}$ is less than $1.5 \mathrm{~ns}$ for Type I diplexer. In the passband, the group delay from input port 1 to output port 2 with the center frequency $4.78 \mathrm{GHz}$ is less than $2.1 \mathrm{~ns}$ and the group delay from input port 1 to output port 3 with the center frequency $5.77 \mathrm{GHz}$ is less than $2.1 \mathrm{~ns}$ for Type II diplexer.

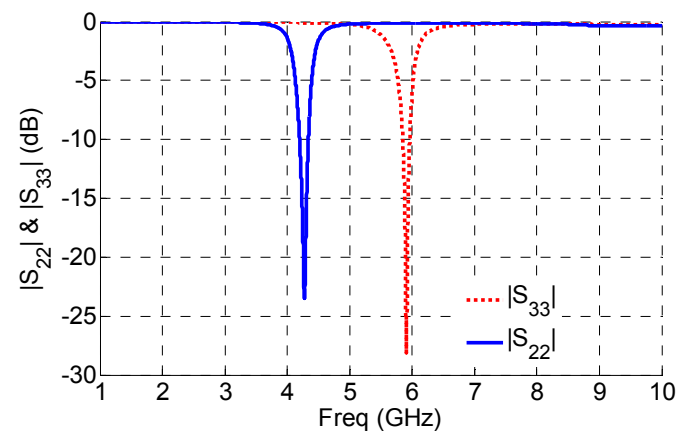

(a)

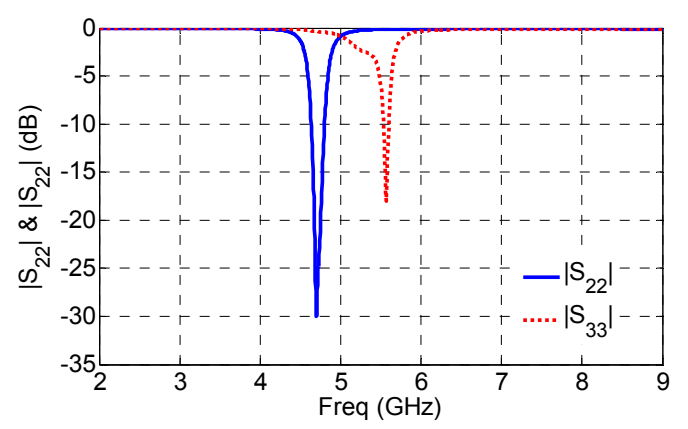

(b)

Fig. 11. Simulated channel filter return losses for ports 2 and 3: (a) Type I, (b) Type II.

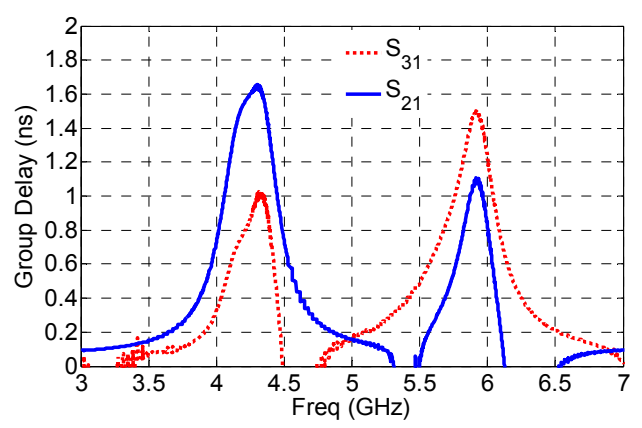

(a)

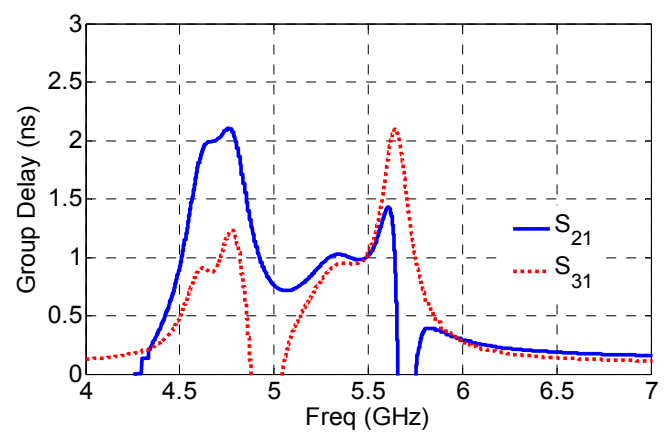

(b)

Fig. 12. (a) Simulated group delays of the Type I at two passbands (maximum group delay variation $0.5 \mathrm{~ns}$ ). (b) Simulated group delays of the Type II at two passbands (maximum group delay variation $0.8 \mathrm{~ns}$ ).

The photograph of the fabricated diplexers are shown in Fig. 13 which demonstrates that the proposed diplexers have very compact size compared with similar diplexers and miniaturization is achieved. Finally, a performance comparison between the proposed diplexers with other 


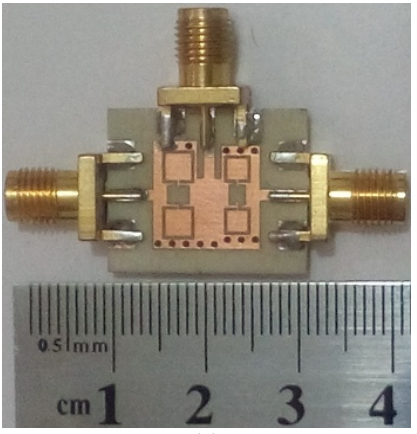

(a)

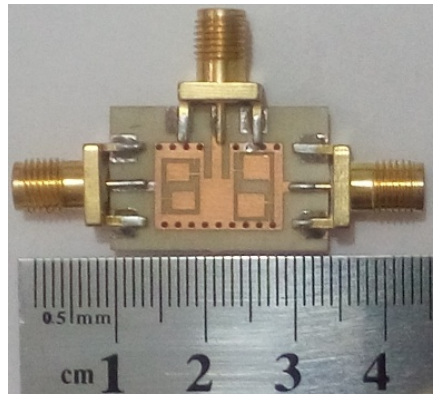

(b)

Fig. 13. Photograph of the fabricated diplexers: (a) Type I, (b) Type II.

reported diplexers which include some miniaturized designs using the microstrip and SIW diplexers are summarizes in Tab. 3. It can be found that the proposed diplexers in this paper have good performances in terms of losses, rejection, isolation, especially size reduction and channel return losses at Port 2 and Port 3.
Generally, the proposed diplexers have many advantages in terms of low insertion loss, high quality factor, easy integration, good passbands performance and most importantly, compact size.

\section{Conclusion}

Two miniaturized SIW diplexers based on the theory of evanescent mode propagation have been designed, fabricated and measured. The proposed SIW diplexers have been realized by cascading two compact SIW-OCSRR filters with different sizes. The OCSRRs behave as an electric dipole and according to the theory of evanescent mode could be used to obtain a forward passband below the waveguide cutoff frequency of the SIW structure. The electrical size of the OCSRRs is larger than that of the CSRRs, therefore this particle is a good candidate to miniaturize the SIW structure. The OCSRRs which have been used in these cases are aligned with face-to-face (Type I) and side-by-side reverse (Type II) orientations. The designed diplexers have advantages like high selectivity, low loss and low cost. High isolation between channels is presented as well. Return losses have been achieved less than $22 \mathrm{~dB}$ and $15 \mathrm{~dB}$ at the lower $(4.33 \mathrm{GHz})$ and higher $(5.99 \mathrm{GHz})$ bands of the Type I diplexer, respectively, and less than $20 \mathrm{~dB}$ and $14 \mathrm{~dB}$ at the lower $(4.78 \mathrm{GHz})$ and higher $(5.77 \mathrm{GHz})$ bands of the Type II diplexer, respectively. The total size of the proposed Type I is less than $0.15 \lambda_{0} \times 0.14 \lambda_{0}$ and the proposed Type II is less than $0.22 \lambda_{0} \times 0.13 \lambda_{0}$ which illustrate the ability of the OCSRR unit cells on the size reduction.

\begin{tabular}{|c|c|c|c|c|c|c|}
\hline \multirow[t]{2}{*}{ Reference number } & \multicolumn{2}{|c|}{$\begin{array}{c}\text { Insertion Loss } \\
\left(\left|\mathbf{S}_{21}\right| \text { or }\left|\mathbf{S}_{31}\right|\right)\end{array}$} & \multicolumn{2}{|c|}{$\begin{array}{c}\text { Suppression } \\
\left(\left|\mathbf{S}_{21}\right| \text { or }\left|\mathbf{S}_{31}\right|\right)\end{array}$} & \multirow{2}{*}{$\begin{array}{c}\text { Size } \\
\left(\lambda_{0} \times \lambda_{0} \times \lambda_{0}\right)\end{array}$} & \multirow{2}{*}{$\begin{array}{l}\text { Isolation } \\
\left(\left|\mathbf{S}_{32}\right|\right)\end{array}$} \\
\hline & Channel 1 & Channel 2 & Channel 1 & Channel 2 & & \\
\hline [6] (part1) & $2.7 \mathrm{~dB}$ & $2.8 \mathrm{~dB}$ & $40 \mathrm{~dB} @ 1.5 \mathrm{GHz}$ & $48 \mathrm{~dB} @ 2.0 \mathrm{GHz}$ & $0.17 \times 0.272 \times 0.003$ & $42 \mathrm{~dB}$ \\
\hline [6] (part2) & $2.8 \mathrm{~dB}$ & $3.2 \mathrm{~dB}$ & $38 \mathrm{~dB} @ 1.5 \mathrm{GHz}$ & $39 \mathrm{~dB} @ 1.76 \mathrm{GHz}$ & $0.187 \times 0.30 \times 0.003$ & $30 \mathrm{~dB}$ \\
\hline$[7]$ & $1.6 \mathrm{~dB}$ & $2.3 \mathrm{~dB}$ & $43 \mathrm{~dB} @ 4.66 \mathrm{GHz}$ & $28 \mathrm{~dB} @ 5.80 \mathrm{GHz}$ & $0.27 \times 0.217 \times 0.008$ & $32 \mathrm{~dB}$ \\
\hline [8] & $1.6 \mathrm{~dB}$ & $2.1 \mathrm{~dB}$ & $42 \mathrm{~dB} @ 9.5 \mathrm{GHz}$ & $37 \mathrm{~dB} @ 10.5 \mathrm{GHz}$ & $2.04 \times 0.65 \times 0.053$ & $35 \mathrm{~dB}$ \\
\hline [9] & $2.2 \mathrm{~dB}$ & $2.4 \mathrm{~dB}$ & $22 \mathrm{~dB} @ 7.75 \mathrm{GHz}$ & $22 \mathrm{~dB} @ 8.25 \mathrm{GHz}$ & $1.44 \times 0.98 \times 0.022$ & - \\
\hline [10] (Diplexer 1) & $1.92 \mathrm{~dB}$ & $2.14 \mathrm{~dB}$ & $33 \mathrm{~dB} @ 7.4 \mathrm{GHz}$ & $42 \mathrm{~dB} @ 8.2 \mathrm{GHz}$ & $3.06 \times 1.38 \times 0.019$ & - \\
\hline [10] (Diplexer 2) & $1.83 \mathrm{~dB}$ & $2.13 \mathrm{~dB}$ & $34 \mathrm{~dB} @ 7.4 \mathrm{GHz}$ & $43 \mathrm{~dB} @ 8.2 \mathrm{GHz}$ & $2.96 \times 1.38 \times 0.019$ & - \\
\hline This work (Type I) & $1.6 \mathrm{~dB}$ & $3.2 \mathrm{~dB}$ & $24 \mathrm{~dB} @ 4.33 \mathrm{GHz}$ & $23 \mathrm{~dB} @ 5.99 \mathrm{GHz}$ & $0.15 \times 0.14 \times 0.007$ & $30 \mathrm{~dB}$ \\
\hline This work (Type II) & $1.8 \mathrm{~dB}$ & $2.3 \mathrm{~dB}$ & $22 \mathrm{~dB} @ 4.78 \mathrm{GHz}$ & $52 \mathrm{~dB} @ 5.77 \mathrm{GHz}$ & $0.22 \times 0.13 \times 0.007$ & $26 \mathrm{~dB}$ \\
\hline
\end{tabular}

Tab. 3. Comparison between the proposed diplexers and the references. $\lambda_{0}$ is the free space wavelength of the first channel center frequency.

\section{References}

[1] BOZZI, M., GEORGIADIS, A., WU, K. Review of substrateintegrated waveguide circuits and antennas. IET Microwaves, Antennas \& Propagation, 2011, vol. 5, no. 8, p. 909-920, DOI: 10.1049/iet-map.2010.0463

[2] MOSCATO, S., TOMASSONI, C., BOZZI, M., PERREGRINI, L. Quarter-mode cavity filters in substrate integrated waveguide technology. IEEE Transactions on Microwave Theory and Techniques, 2016, vol. 64, no. 8, p. 2538-2547. DOI: 10.1109/TMTT.2016.2577690

[3] CHEN, X., WU, K. Substrate integrated waveguide filters: Design techniques and structure innovations. IEEE Microwave Magazine,
2014, vol. 15, no. 6, p. 121-133, DOI: 10.1109/MMM.2014.2332886

[4] GARG, R., BAHL, I., BOZZI, M. Microstrip Lines and Slotlines. $3^{\text {rd }}$ ed. Artech House, 2013. ISBN: 9781608075355

[5] BONACHE, J., GIL, I., GARCA-GARCIA, J., MARTIN, F. Complementary split ring resonators for microstrip diplexer design. Electronics Letters, 2005, vol. 41, no. 14, p. 810-811. DOI: $10.1049 / \mathrm{el}: 20050895$

[6] CHEN, C.-H., HUANG, T.Y., CHOU, C.P., WU, R.B., Microstrip diplexers design with common resonator sections for compact size but high isolation. IEEE Transactions on Microwave Theory and Techniques, 2006, vol. 54, no. 5, p. 1945-1952. DOI: 10.1109/TMTT.2006.873613 
[7] DONG, Y., ITOH, T. Substrate integrated waveguide loaded by complementary split-ring resonators for miniaturized diplexer design. IEEE Microwave Wireless Component Letters, 2011, vol. 21, no. 1, p. 10-12. DOI: 10.1109/LMWC.2010.2091263

[8] SIRCI, S., MARTINEZ, J. D., VAGUE, J., BORIA, V. E. Substrate integrated waveguide diplexer based on circular triplet combline filters. IEEE Microwave and Wireless Components Letters, 2015, vol. 25, no. 7, p. 430-432. DOI: 10.1109/LMWC.2015.2427516

[9] GARCIA-LAMPEREZ, A., SALAZAR-PALMA, M., YEUNG, S.-H. SIW compact diplexer. In IEEE MTT-S International Microwave Symposium Digest. Tampa Bay (FL, USA), Jun. 2014 p. 1-4. DOI: 10.1109/MWSYM.2014.6848514

[10] ZHAO, C., Z., FUMEAUX, C., LIM, C-C. Substrate-integrated waveguide diplexers with improved Y-junctions. Microwave and Optical Technology Letters, 2016, vol. 58, no. 6, p. 1384-1388. DOI: $10.1002 /$ mop.29807

[11] CALOZ, C., ITOH, T. Electromagnetic Metamaterials: Transmission Line Theory and Microwave Applications. New York (USA): John Wiley \& Sons, 2006. DOI: 10.1002/0471754323

[12] MARQUES, R., MARTIN, F., SOROLLA, M. Metamaterials with Negative Parameters: Theory, Design and Microwave Applications. New York (USA): John Wiley \& Sons, 2011. ISBN: 9781118211564

[13] FERRAN, M. Artificial Transmission Lines for RF and Microwave Applications. John Wiley, 2015. ISBN: 978-1-118-48760-0

[14] DONG, Y., YANG, T., ITOH, T. Substrate integrated waveguide loaded by complementary split-ring resonators and its applications to miniaturized waveguide filters. IEEE Transactions on Microwave Theory and Techniques, 2009, vol. 57, no. 9, p. 2211-2223. DOI: 10.1109/TMTT.2009.2027156

[15] DANAEIAN, M., AFROOZ, K., HAKIMI, A., MOZNEBI, A.-R., Compact bandpass filter based on SIW loaded by open complementary split-ring resonators (OCSRRs). International Journal of $R F$ and Microwave Computer-Aided Engineering, 2016, vol. 26, no. 8, p. 674-682. DOI: $10.1002 /$ mmce. 21017

\section{About the Authors ...}

Mostafa DANAEIAN was born in Yazd, Iran, in 1985. He received the B.Sc. degree in Electrical Engineering from Yazd University, Yazd, Iran, in 2008, the M.S. degree in Electrical Engineering from Shahid Bahonar University of Kerman, Kerman, Iran, in 2011, and is currently working toward the Ph.D. degree at the Shahid Bahonar University of Kerman, Kerman, Iran. His research interests are MTM, $\mathrm{RF} /$ microwave circuits design. He is currently involved with microwave devices based on SIW structures.

Kambiz AFROOZ was born in Baft, Iran, in 1983. He received the B.Sc. degree from the Shahid Bahonar University of Kerman, Kerman, Iran, in 2005, the M.Sc. and Ph.D. degrees from the Amirkabir University of Technology (Tehran Polytechnic), Tehran, Iran, in 2007 and 2012, all in Electrical Engineering. In May 2011, he joined the CIMITEC group, University Autonoma de Barcelona (UAB), Barcelona, Spain, as a Visiting Student. He is currently an Associate professor with the Electrical Engineering Department, Shahid Bahonar University of Kerman, Kerman, Iran. His research interests are in the areas of computer-aided design of active and passive microwave devices and circuits, computational electromagnetic, metamaterial transmission lines, and substrate integrated waveguide structures.

Ahmad HAKIMI was born in Rafsanjan, Iran, in 1961. He received his B.Sc. degree in Electrical Engineering from the Technical College of Shahid Bahonar University of Kerman, Kerman, Iran, in 1986. Using the scholarship granted by the Ministry of Higher Education of Iran and Istanbul Technical University (ITU) in 1987, he has been studying for his M.Sc. and Ph.D. degrees in the Faculty of Electrical and Electronic Engineering at the ITU. He received his M.Sc. and Ph.D. degrees from ITU in 1996 and 1995 in the field of high frequency electronics. His research interests include design and analysis of nonlinear RF circuits, numerical analysis and advanced engineering mathematics, analog filter design, and linear integrated circuits. 